\section{Security that can't be beaten}

Digital information is under threat in the modern age with emails being hacked and social media not providing adequate protection, according to PRO Diagnostics UK.

The company says that when it comes to sensitive patient information you need to be sure you have a secure way to store it that can be trusted and PROPACS from PRO Diagnostics UK is the solution.

An innovative cloud-based image storage system, PROPACS features highly advanced encrypted software that protects sensitive information from outside threats, safeguarding it from any potential risks such as hackers.

As well as this exceptional level of protection, PROPACS can also be used as a diagnostic radiograph reporting system.

Professionals with a PROPACS account can access their on-line diagnostic back up files and request to have the individual study evaluated by specialist dental radiologists for a fully detailed report. These reports take just 24 hours to be turned around, providing all the necessary information for a fast and truly effective care plan.

More information can be found by visiting www.prodiagnostics.co.uk or emailing sales@prodiagnostics.co.uk.

\section{The secret to a new smile}

Orthodontic solutions don't have to be unsightly - introduce your patients to the discreet way to straighten their smile with the Incognito system from 3M Oral Care.

Designed to help orthodontists meet the demand for more aesthetic solutions, the Incognito appliance system is fully customised to each patient, making it simple to treat many different case types. Furthermore, due to its precise slot positioning the system expresses torque effectively, ${ }^{1}$ giving patients the results they require without compromising their confidence during treatment.

Share the secret behind ultimate discreet orthodontics by contacting the team at $3 \mathrm{M}$ Oral Care for more information.

For more information, call 08458734066 or visit http:// solutions.3m.co.uk/wps/portal/3M/en_GB/orthodontics_EU/ Unitek/.

1. 3 M Unitek data on file, 3M Unitek Claim DB Centre 'Incognito Appliance System: A clinical study illustrates the Incognito System slots are significantly more precise than others, leading to the ability to express torque efficiently.' Claim Number: 2012 - S0

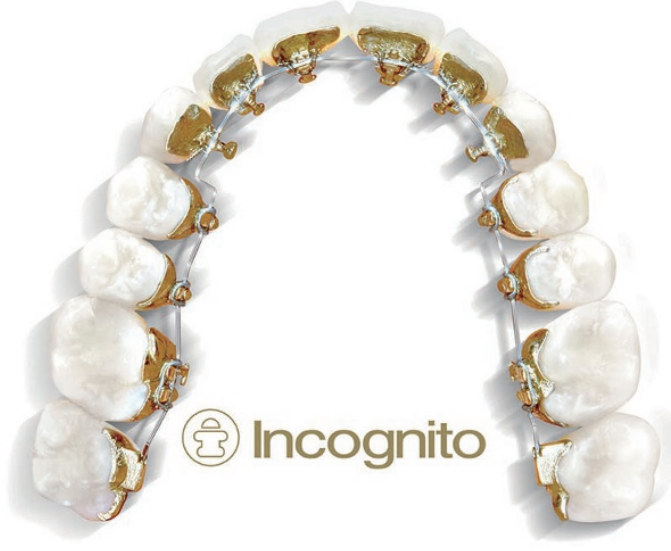

\section{Support you can rely on}

When providing patients with NHS treatments, dentists need a laboratory that can understand the demands that these services entail.

Dentists can ensure they have technical support

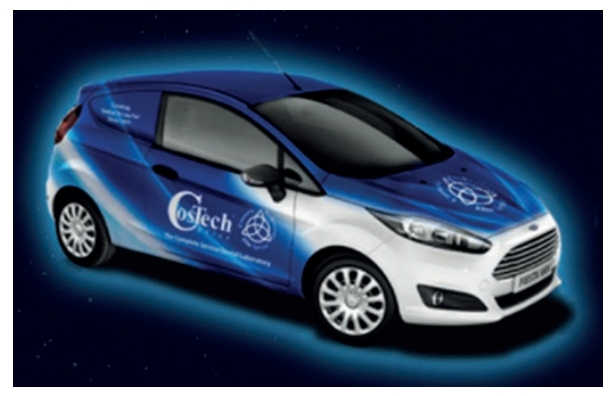
they can rely on by choosing CosTech Dental Laboratory.

A trusted choice since 1977, CosTech began life as a specialist crown and bridge service and has a vital understanding of how NHS dentistry works. Today, the team still has dedicated bridge and crown technicians in-house who can provide dedicated NHS specific services.

All restorations from CosTech are completed on articulated models and provide a guaranteed fit on solid models, helping customers to ensure that the best fit and accuracy is achieved.

Furthermore, CosTech offers free delivery on all cases coming to and from the laboratory and competitive pricing on standard NHS treatment options such as crowns and bridges. CosTech is DAMAS certified as a trusted supplier of high quality products.

More information about CosTech Dental Laboratory is available by visiting www.costech.co.uk or calling 01474320076 .

\section{Effective implant imaging}

Digital imaging has become an essential aspect of successful dental treatment, ensuring accurate diagnostics and treatment planning for optimised outcomes.

The CS 8100 3D imaging unit from Carestream Dental is the ideal solution for practices of all sizes with its compact design and affordable price bringing the benefits of $3 \mathrm{D}$ imaging within the reach of every clinician.

Offering panoramic and 3D imaging, with optional cephalometric capabilities, the CS 8100 3D produces sharp, contrasted images with a resolution of up to $75 \mu \mathrm{m}$. You can view areas of interest from any angle with a one-to-one accuracy and no overlap or distortion.

With the added convenience of being able to adjust the machine to suit patients when standing or sitting, the open, faceto-face design and fast scanning time promote patient comfort and reduce the need for retakes.

More information is available by contacting Carestream Dental on 08001699692 or by visiting www.carestreamdental.co.uk.

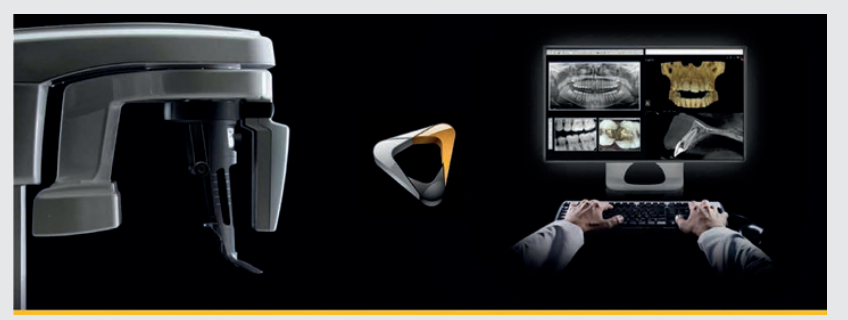

